\title{
Multi-flux-transformer MRI detection with an atomic magnetometer
} Igor Savukov ${ }^{1}$ and Todor Karaulanov ${ }^{2}$ - Los Alamos National Laboratory, Los Alamos, NM 87545, USA

\begin{abstract}
Recently, anatomical ultra-low field (ULF) MRI has been demonstrated with anatomic magnetometer (AM). A flux-transformer (FT) has been used for decoupling MRI fields and gradients to avoid their negative effects on AM performance. The field of view (FOV) was limited because of the need to compromise between the size of the FT input coil and MRI sensitivity per voxel. Multi-channel acquisition is a well-known solution to increase FOV without significantly reducing sensitivity. In this paper, we demonstrate two-fold FOV increase with the use of three FT input coils. We also show that it is possible to use a single atomic magnetometer and single acquisition channel to acquire three independent MRI signals by applying a frequency-encoding gradient along the direction of the detection array span. The approach can be generalized to more channels and can be critical for imaging applications of non-cryogenic ULF MRI where FOV needs to be large, including head, hand, spine, and whole-body imaging.
\end{abstract}

\section{Introduction}

Ultra-low field (ULF) MRI can be a valuable alternative to high-field MRI with the promise of cost reduction and portability [1], high tissue contrast[2], absence of susceptibility artifacts [3], and the possibility to combine MRI with other modalities, such as magneto-encephalography [4]. ULF brain imaging has already been demonstrated [4]. It has the potential to be used in some low-resolution practical applications, such as the detection of large brain tumors or diagnosis of severe brain injuries, but the image quality needs to be improved for most clinical applications. The inferior image quality is a direct consequence of inherently low signal-to-noise ratio (SNR) and artefacts introduced by the use of strong pulsed pre-polarization fields. In order to obtain a useful image, the most sensitive magnetic sensors, such as super conductive quantum interference devices (SQUIDs) inside a shielded room, and multi-sensor parallel imaging have been used in ULF-MRI systems [4,5]. Unfortunately, SQUIDs introduce problems associated with cryogenic operation, and the need of shielded rooms increased the price and weight of the system.

The need for cryogens can be avoided with atomic magnetometers (AMs). AMs have demonstrated femtotesla sensitivity [6,7], similar to SQUIDs, and low-cost radio-frequency (RF) AM sensors have been designed and tested [8]. The SQUID-like sensitivity of AMs has also been demonstrated in MRI[9]. Furthermore, from the fundamental AM noise analysis [10], it follows that the sub-femtotesla sensitivity could bring ULF MRI closer to practical needs of clinical diagnostics. However, there are disadvantages of

\footnotetext{
${ }^{1}$ Corresponding author -- I. Savukov: isavukov@lanl.gov, Tel. 505-667-7659, Fax 505-665-2549

${ }^{2}$ Present affiliation of T. Karaulanov: Senior Scientific LLC, Albuquerque, NM 87106
} 
AM in MRI applications as well: a particularly important one is the sensitivity of the AM to magnetic fields and gradients that are present during the detection phase in MRI.

Various approaches to decouple the MRI field from AM such as remote detection [11], solenoid-based field separation [12-14], and flux transformer (FT) mediated detection $[9,15,16]$ have been proposed. The FT-mediated detection method is currently the simplest and most convenient method of field and gradient decoupling that is fully compatible with anatomical imaging. Important additional advantages of the method are: simplifications in the shield design and reduction of its size; the possibility of simple gradiometric noise subtraction; the possibility to minimize interference from the excitation coil; compact AM design with a separate small shield; increased bandwidth without loss of sensitivity; decoupling of low-frequency noise components. However, there is also one important drawback: the room-temperature FT introduces Johnson noise, which affects the SNR. Fortunately, this noise can be reduced by increasing the detection frequency. At $100 \mathrm{kHz}$, in particular, the sensitivity becomes sufficient for rudimental anatomical imaging, including the imaging of the human brain [17]. The limitation of Johnson noise also raises the question about comparison of the FT-AM detection method with the more conventional coil-amplifier detection method. The main advantage, as we discussed it previously [15], is the ability to reduce coupling between channels to improve the sensitivity of the multi-channel detection. This will be particularly important when the sensitivity and the number of channels will be increased in the future for improving image quality and increasing FOV to approach the performance of other scanners.

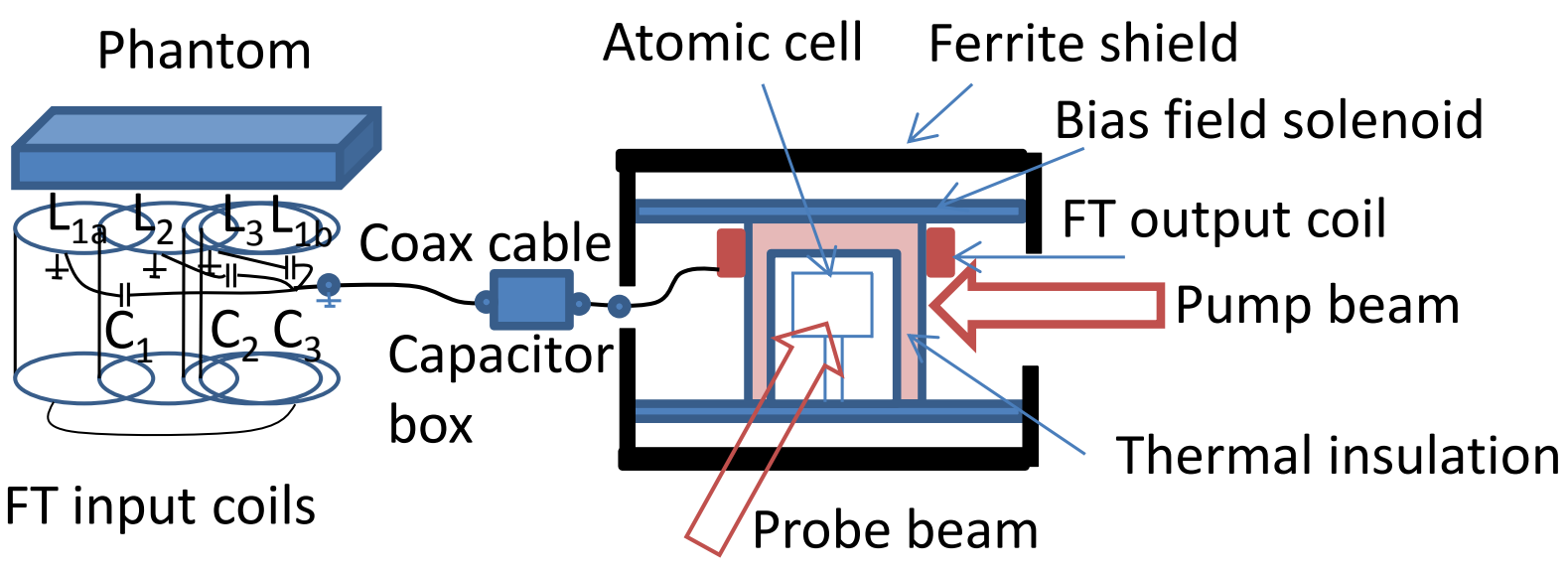

Fig.1. Multi-flux-transformer AM detection system.

${ }^{1}$ Corresponding author -- I. Savukov: isavukov@lanl.gov, Tel. 505-667-7659, Fax 505-665-2549

${ }^{2}$ Present affiliation of T. Karaulanov: Senior Scientific LLC, Albuquerque, NM 87106 


\section{Method and Experimental Results}

\section{Multi-flux-transformer detection}

Multi-channel detection is a method to increase SNR, accelerate the MRI scan, reduce required bandwidth per sensor, and increase the field of view (FOV) [5, 18-21]. For example, if one large coil is replaced with $\mathrm{N}$ small coils covering the same area and the imaging depth is less than the radius of the small coil, from the principle of reciprocity, which states that the NMR signal from a voxel is proportional to the field produced by the detection coil at the position of the voxel, an increase in the signal is expected by a factor of Sqrt[N]. At the same time the coils' noise is also reduced since smaller coils have lower intrinsic Johnson noise (e.g. due to shorter length of the wire) and are less sensitive to the external interferences (the voltage from environmental field noise will decrease linearly with the area of

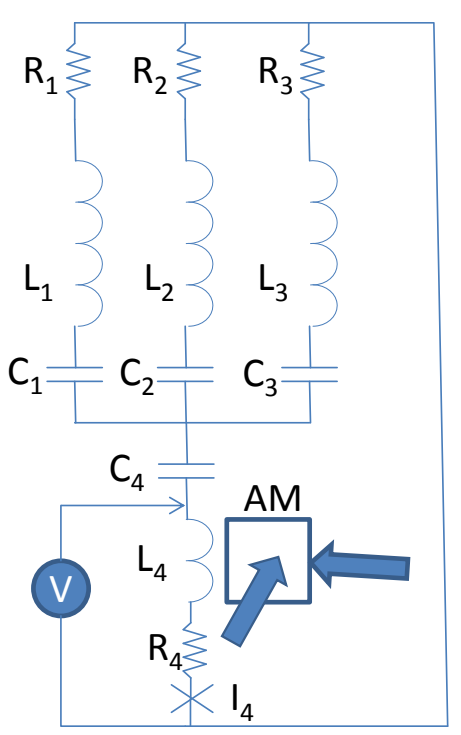

Fig. 2. Three-channel FT-AM detection diagram with one shared AM. Resistances $R_{1}-R_{3}$ account for finite quality factors of the coils $L_{1}-L_{3}$. Capacitors $C_{1}-C_{4}$ serve to enhance the efficiency of the field transfer. Tuning of the circuit can be performed by measuring voltage as indicated. The cross indicates the current measurement point that can be also used for tuning instead of the AM. the coil). This further improves the gain in SNR. Since the SNR level is quite low in the ULF MRI regime, the multichannel detection is highly desirable. The multi-channel detection can be arranged for imaging the whole body as well as its parts. A brain scan in [17] took thirteen minutes and covered a part of the brain about $4 \mathrm{~cm}$ deep and 10 $\mathrm{cm}$ wide. To acquire the whole brain image with the same MRI parameters (similar prepolarization and measurement fields), it would be necessary to implement 4-8 detection channels. For hand [9] and forearm [22] imaging, multiple channels will help to reach $1 \mathrm{~mm}$ resolution. Perhaps, the most beneficial and easy to implement would be a 1D array for imaging the spine. These and some other imaging applications strongly motivate the development of multichannel approach with FT-AM detectors.

The multi-flux transformer detection system with AM is depicted in

Fig. 1. It follows the idea of a single-channel FT-AM system previously described in $[9,15,17]$, but the signals from multiple input coils $\left(L_{1 a}, L_{2}, L_{3}\right.$ are actually gradiometers each consisting of two Litz-wire 35-turn coils

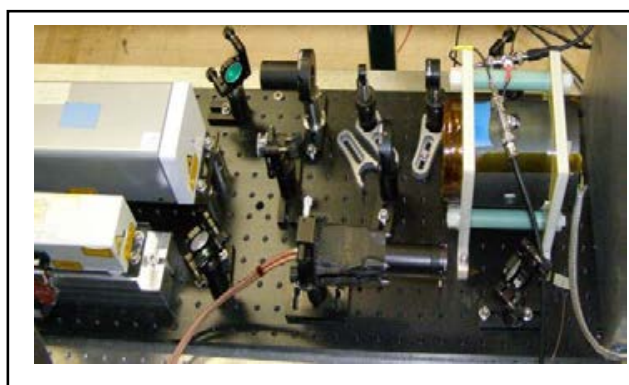

Fig. 3. Photo of atomic magnetometer setup

${ }^{1}$ Corresponding author -- I. Savukov: isavukov@lanl.gov, Tel. 505-667-7659, Fax 505-665-2549

${ }^{2}$ Present affiliation of T. Karaulanov: Senior Scientific LLC, Albuquerque, NM 87106 
connected in the opposite direction; $\mathrm{L}_{1}=\mathrm{L}_{1 \mathrm{a}}+\mathrm{L}_{1 b}$, where $\mathrm{L}_{1 \mathrm{~b}}$ is a 3-turn compensation coil; diameters of all coils are $6 \mathrm{~cm}$ ) are combined in one output coil as in Fig. 2 . The capacitors are added to tune $L_{1} C_{1}, L_{2} C_{2}$, $\mathrm{L}_{3} \mathrm{C}_{3}$ to slightly different resonance frequencies, and $\mathrm{C}_{4}$ to compensate the impedance of the output coil $\mathrm{L}_{4}$. The input coils are located in the MRI region to pick up the NMR magnetic signal, and the output coil is located inside a compact ferromagnetic shield where its field is detected with an AM. With this arrangement, no large high-mu shielding structure is required. To reduce the magnetic noise inside the MRI coil system, the input coils are configured as first-order gradiometers (Fig 1 top and bottom coils).

The AM, in Fig. 1 shown inside the ferrite shield, consists of an Atomic cell, a Pump beam, and a Probe beam. The circularly polarized Pump beam is used to orient atomic spins along its direction, and the linearly polarized Probe beam is used to read-out the atomic spin states. The Pump and Probe beams intersect inside the Atomic cell at $90^{\circ}$, defining the active volume of the AM. The optics and lasers producing the Pump and Probe beams are mounted on an aluminum breadboard (Fig. 3). In more detail the AM used in the current experiments was described in Ref.[17]. More information about AM

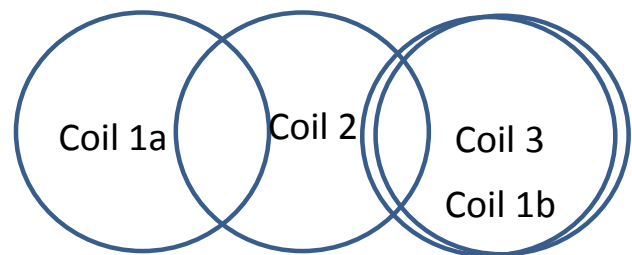

Fig. 4. Geometrical arrangement of three upper FT input coils minimizing mutual fluxes. construction and operation can be found in Refs. $[10,14,15]$.

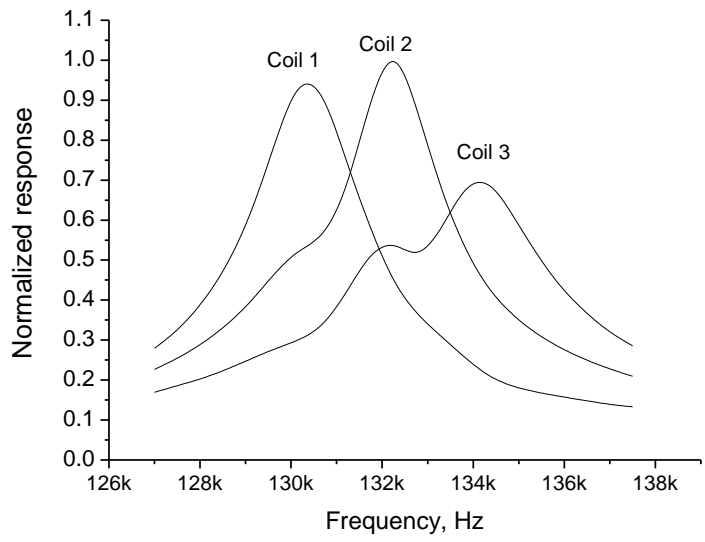

Fig. 5. Frequency response of the detection circuit shown in Fig. 2 inside the MRI bore when a small coil is placed near the center of each FT input coil to generate localized RF magnetic field. Three distinct resonances are observed as expected when the channels operate independently. The doublepeak structure of the response of Coil 3 indicates some small residual coupling. It appeared when the system was placed inside the bore.
To realize independent channels, it is important to mutually decouple input coils. This can be done by adjusting positions of the coils with respect to each other and by deforming them while monitoring coils outputs from an applied sine signal. Since we have input coils configured as gradiometers, the decoupling has been done for both the upper and the lower coils of the gradiometers. The upper coils have been decoupled first. The sequence of steps is the following: 1 ) decouple the upper Coil1a and the upper Coil2 by moving the upper Coil 1a (Fig.4); 2) decouple the upper Coil 2 and the upper Coil 3 by moving the upper Coil 3; 3) adjust the position of the upper compensation coil (Coil 1b, connected to Coil 1a to form Coil 1) to minimize flux between the upper Coil 1 and the upper Coil 3; 4) minimize coupling between all 3 upper coils by deforming slightly (a few .gov, Tel. 505-667-7659, Fax 505-665-2549

\footnotetext{
${ }^{2}$ Present affiliation of T. Karaulanov: Senior Scientific LLC, Albuquerque, NM 87106
} 
$\mathrm{mm}$ ) all the upper coils; 5) conduct the same procedure for the lower coils; 6) connect the upper and lower coils as gradiometers; 7) slightly deform all coils for fine minimization of coupling between 3 input gradiometers. This procedure is justified by the result that the residual coupling has been reached at $1 \%$ level. After finishing decoupling, the coils have been fixed rigidly with glue and tape. We have decoupled channels when the coils were outside of the MRI system, but we found that the coupling slightly increased upon inserting the FT coil system into the MRI bore.

The above-described decoupling procedure can be generalized to a larger number of coils; however, decoupling becomes progressively more difficult and is affected by surrounding conductive objects. Use of atomic magnetometers reduces requirement on the decoupling level and simplifies the tuning of the system.

When imaging of a long object is needed, a frequency encoding gradient can be applied along the length of the object and instead of multiple atomic magnetometers a single AM can be used connected according to the diagram in Fig. 2. The channels' independent operation is ensured by frequency spacing of responses of different coils. Indeed, experimentally we observed that when a small coil was positioned in the center of each of three input FT coils connected as in Fig.2, the response of each coil was almost

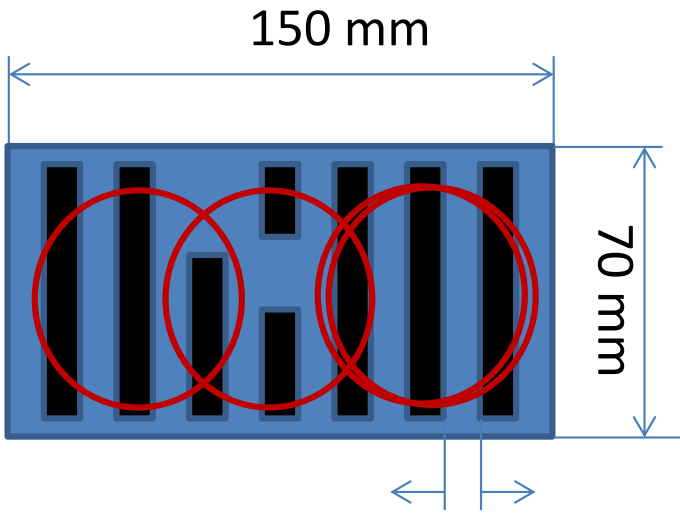

$10 \mathrm{~mm}$ independent of the other two (Fig.4) . This requires certain mutual detuning of the coils. If resonances are detuned enough, as in Fig. 5, only noise from a single coil will be dominating near its resonance.

Unfortunately, good decoupling in our experiment for specific $Q$ factors of the coils was achieved with the spacing $2 \mathrm{kHz}$ between resonances, so it was necessary to tune the $A M$ to three different frequencies during the run instead of using a single tuning setting. With higher $Q$, smaller residual coupling, and a more efficient output transformer $\left(\mathrm{L}_{4}\right.$ output coil matching the size of the AM cell and positioned closely to the cell), a single AM bias field can be used to realize simultaneous parallel MRI.

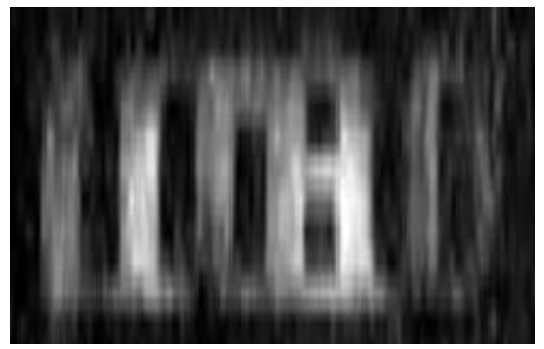

Fig. 6. Imaging the phantom with the 3channel system. The demonstrated FOV is $130 \mathrm{~mm}$, which is expected for our 3-coil system and substantially exceed that of a single coil. The blue region is filled with $\mathrm{CuSO}_{4}$ solution and corresponds to the bright regions in MRI.

${ }^{1}$ Corresponding author -- I. Savukov: isavukov@lanl.gov, Tel. 505-667-7659, Fax 505-665-2549

${ }^{2}$ Present affiliation of T. Karaulanov: Senior Scientific LLC, Albuquerque, NM 87106 


\section{MRI phantom experiment}

Using the three-channel FT+AM system we conducted MRI phantom experiments to demonstrate the increase of FOV over that of a single-channel system. The MRI hardware, software, and pulse sequence have been described in detail in our previous paper [17] and the references therein. Briefly, initially a pre-poarlization field $\mathrm{Bp}$ of $100 \mathrm{mT}$ is turned on to polarize proton spins and turned off during ultra-low field measurements at $130 \mathrm{kHz}$. The NMR signal is excited with $\mathrm{Pi} / 2$ pulses slightly delayed after Bp off to minimize the effects of $\mathrm{Bp}$ transients, and $\mathrm{Pi}$ pulse is applied to rephase spins at the echo time. A constant frequency encoding and two pulsed phase-encoding gradients are applied between the $\mathrm{Pi} / 2$ and $\mathrm{Pi}$ pulses to realize 3D imaging. The sequence is repeated multiple times to cover required phase space. The imaging sequence parameters were chosen to give approximately $3 \times 3 \mathrm{~mm}^{2}$ in-plane resolution. The same potassium atomic magnetometer was used as in [17]. The atomic cell was heated to $180^{\circ} \mathrm{C}$. The bandwidth was intentionally increased by using larger than optimal pump laser power. This reduced AM sensitivity; however, the loss of sensitivity was compensated by near-resonance enhancement of the circuit (Fig. 2).

An imaging phantom was inserted in the middle of the bore. The phantom pattern and dimensions are shown in Fig. 6 (top). The specific pattern was designed to be able to distinguish symmetric orientations and identify imaged parts of the phantom. The three FT input coils (red circles) were located with respect to the phantom as depicted. The phantom contained copper sulfate solution with $\mathrm{T}_{1}$ of $300 \mathrm{~ms}$.

The images from 3 channels were combined in intensity. The resulting image is shown in Fig. 6 (bottom). It is clear that the three-channel system substantially increased FOV, from $60 \mathrm{~mm}$ (the diameter of the coil) to $130 \mathrm{~mm}$. The coil decoupling overlap limited the largest achievable FOV. The rightmost portion of the image has lower SNR due to some loss of sensitivity from residual coupling, as can be anticipated from the larger peak width of Coil 3 in Fig. 5. Thus it is indeed important to carefully decouple coils.

\section{Discussion and conclusion}

Ultra-low field MRI is a promising method with various advantages, such as low cost and portability. Low SNR is the main drawback and multi-channel operation is essential for improving sensitivity and image quality. Cryogenic operation of SQUID-based systems can be avoided with the use of AMs. The FT-AM approach is suitable for anatomical imaging, as previously shown, and for multi-channel operation as we demonstrated here. Although we used three FTs to achieve two-fold FOV increase, the approach can be generalized to include a larger number of channels. Along the frequency-encoding direction channels can be either coupled to a single broad-band AM or to multiple atomic magnetometers with narrower bandwidth. Because the magnetometer loses sensitivity when its spectrum is broadened, the observed image SNR is lower than in previous work. To amend this, it will be necessary to reduce the size of the AM cell and bring the FT output coil closer to the cell [16]. The temperature of the cell can also be increased (the current oven design did not allow such increase) to improve sensitivity for larger

${ }^{1}$ Corresponding author -- I. Savukov: isavukov@lanl.gov, Tel. 505-667-7659, Fax 505-665-2549

${ }^{2}$ Present affiliation of T. Karaulanov: Senior Scientific LLC, Albuquerque, NM 87106 
bandwidth. To extend the FOV in two directions, the multi-channel operation can be additionally realized in a phase-encoding direction. For this multiple AMs will be required.

\section{Acknowledgement}

This work is sponsored by NIH Grant 5 R01 EB009355. The work of T. Karaulanov was partially supported by the U.S. Department of Energy through the LANL/LDRD Program.

\section{Literature}

1. A. Macovski, S. Conolly, Novel Approaches to Low-Cost MRI, Magnetic Resonance in Medicine 40 (1993) 221-230.

2. J. Clarke, M. Hatridge, M. Mößle, SQUID-Detected Magnetic Resonance Imaging in Microtesla Fields, Annu. Rev. Biomed. Eng. 9 (2007) 389-413.

3. M. Mössle, S. Han, W.R. Myers, S.K. Lee, N. Kelso, M. Hatridge, A. Pines, J. Clarke, SQUID-detected microtesla MRI in the presence of metal, JMR 179 (2006)_146-51.

4. V.S. Zotev, A.N. Matlashov, P.L. Volegov, I.M. Savukov, M.A. Espy, J.C. Mosher, J.J. Gomez, R.H. Kraus Jr. Microtesla MRI of the human brain combined with MEG, JMR 194 (2008) 115-120.

5. V.S. Zotev, P.L. Volegov, A.N. Matlashov, M.A. Espy, J.C. Mosher, R.H. Kraus Jr., Parallel MRI at microtesla fields," JMR 192 (2008) 197-208.

6. J.C. Allred, R.N. Lyman, T.W. Kornack, M.V. Romalis, High-Sensitivity Atomic Magnetometer Unaffected by Spin-Exchange Relaxation, Phys. Rev. Lett. 89 (2002) 130801.

7. I.K. Kominis, T.W. Kornack, J.C. Allred, M.V. Romalis, A subfemtotesla multichannel atomic magnetometer, Nature 422 (2003) 596.

8. I. Savukov, T. Karaulanov, M.G. Boshier, Ultra-sensitive high-density Rb-87 radio-frequency magnetometer, Appl. Phys. Lett. 104 (2014) 023504.

9. I. Savukov, T. Karaulanov, Anatomical MRI with an atomic magnetometer, JMR 231(2013) 39-45.

10. I.M. Savukov, S.J. Seltzer, M.V. Romalis, K.L. Sauer, Tunable Atomic Magnetometer for Detection of RadioFrequency Magnetic Fields, Phys. Rev. Lett. 95(2005) 063004.

11. S. Xu, V.V. Yashchuk, M.H. Donaldson, S.M. Rochester, D. Budker, A. Pines, Magnetic resonance imaging with an optical atomic magnetometer, PNAS 103 (2006) 12668-12671.

12. I.M. Savukov, M.V. Romalis, NMR Detection with an Atomic Magnetometer, Phys. Rev. Lett. 94 (2005) 123001.

13. V. V. Yashchuk, J. Granwehr, D. F. Kimball, S. M. Rochester, A. H. Trabesinger, J. T. Urban, D. Budker, and A. Pines, Hyperpolarized Xenon Nuclear Spins Detected by Optical Atomic Magnetometry, Phys. Rev. Lett. 93 (2004) 160801.

14. I.M. Savukov, S.J. Seltzer, and M.V. Romalis, Detection of NMR signals with a radio-frequency atomic magnetometer, JMR 185 (2007) 214-20.

${ }^{1}$ Corresponding author -- I. Savukov: isavukov@lanl.gov, Tel. 505-667-7659, Fax 505-665-2549

${ }^{2}$ Present affiliation of T. Karaulanov: Senior Scientific LLC, Albuquerque, NM 87106 
15. I.M. Savukov, V.S. Zotev, P.L. Volegov, M.A. Espy, A.N. Matlashov, J.J. Gomez, R.H. Kraus, Jr., MRI with an atomic magnetometer suitable for practical imaging applications, JMR 199 (2009) 188-191.

16. T. Oida, Y. Kawamura, T. Kobayashi, Optimization of Flux Transformer for Optically Pumped Atomic Magnetometer in Ultra-Low Field MRI Systems, IEEE Transections on magnetics, 47 (2011) 3074.

17. I. Savukov and T. Karaulanov, Magnetic-resonance imaging of the human brain with an atomic magnetometer, Appl. Phys. Lett. 103 (2013) 043703.

18. J.S. Hyde, A. Jesmanowicz, W. Froncisz, J.B. Kneeland, T.M. Grist, N.F. Campagna, Parallel image cquisition from noninteracting local coils, J. Magn. Reson. 70 (1986) 512-517.

19. P.B. Roemer, W.A. Edelstein, C.E. Hayes, S.P. Souza, O.M. Mueller, The NMR phased array, Magn. Reson. Med. 16 (1990) 192-225.

20. L.L. Wald, L. Carvajal, S.E. Moyher, S.J. Nelson, P.E. Grant, A.J. Barkovich, D.B. Vigneron, Phased array detectors and an automated intensity-correction algorithm for high-resolution MR imaging of the human brain, Magn. Reson. Med. 34 (1995) 433-439.

21. S.M. Wright, L.L. Wald, Theory and application of array coils in MR spectroscopy, NMR Biomed. 10 (1997) 394-410.

22. I. Savukov T. Karaulanov C.J.V. Wurden L. Schultz, Non-cryogenic ultra-low field MRI of wrist-forearm area, JMR 233 (2013) 103-106

${ }^{1}$ Corresponding author -- I. Savukov: isavukov@lanl.gov, Tel. 505-667-7659, Fax 505-665-2549

${ }^{2}$ Present affiliation of T. Karaulanov: Senior Scientific LLC, Albuquerque, NM 87106 


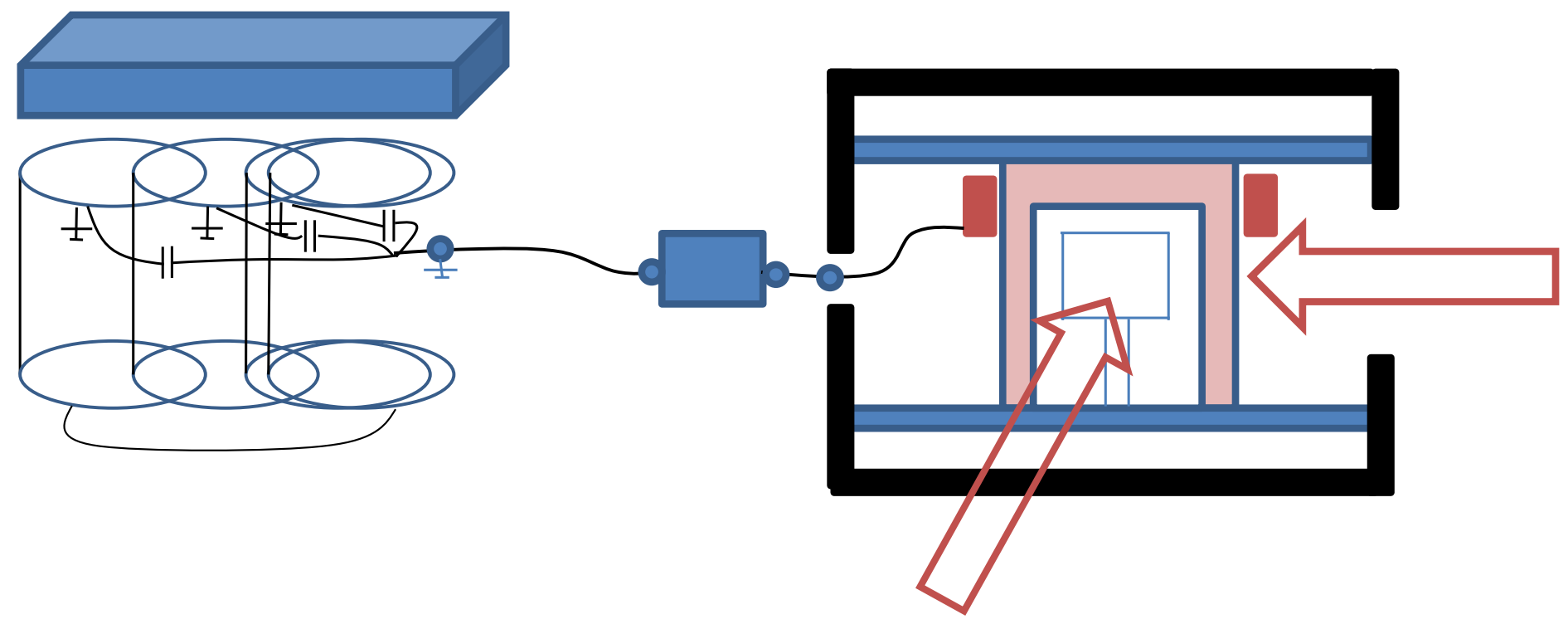

